\title{
The predictors and prognosis for unexpected reocclusion after mechanical thrombectomy: a meta-analysis
}

\author{
Xiangyu Li ${ }^{1 \#}$, Furong Gu ${ }^{1 \#}$, Jiayue Ding ${ }^{2}, \mathrm{Ji} \mathrm{Bian}^{1}, \mathrm{Na} \mathrm{Wang}^{1}$, Rui Shu ${ }^{1}$, Qingyun $\mathrm{Li}^{1}$, Xiaolin Xu ${ }^{1}$ \\ ${ }^{1}$ Department of Neurology, Tianjin Huanhu Hospital, Tianjin, China; ${ }^{2}$ Department of Neurology, Tianjin Medical University General Hospital, \\ Tianjin, China \\ Contributions: (I) Conception and design: X Li, F Gu, J Ding, X Xu; (II) Provision of study materials or patients: X Li, J Ding, J Bian, R Shu, N \\ Wang; (III) Collection and assembly of data: X Li, J Ding, J Bian, R Shu, N Wang; (IV) Data analysis and interpretation: J Ding, F Gu, Q Li; (V) \\ Manuscript writing: All authors; (VI) Final approval of manuscript: All authors. \\ "These authors contributed equally to this work. \\ Correspondence to: Xiaolin Xu, MD, PhD. Department of Neurology, Tianjin Huanhu Hospital, Tianjin, China. Email: hhyyxxl@163.com.
}

Background: Mechanical thrombectomy (MT) is the cornerstone for treating acute ischemic stroke (AIS) in emergency cases. However, 3-9\% of patients display reocclusion in the recanalized vessels within 24 hours after performing MT. This meta-analysis aimed to further identify the predictors and prognosis of unexpected reocclusion after MT.

Methods: According to the Preferred Reporting Items for Systematic Reviews and Meta-Analyses statement, we searched several literature databases, including PubMed, Embase, and Cochrane, for publications related to the subject term "thrombectomy" that were published prior to March 2020. Pooled analysis was performed with the fixed-effects model using the Mantel-Haenszel method if the heterogeneity was expected to be available $\left(\mathrm{I}^{2} \leq 50 \%\right)$. Otherwise, the random-effects model computed by the DerSimonianLaird method was used ( $\mathrm{I}^{2}>50 \%$ ). R software (http://www.r-project.org) was used for analysis in this study.

Results: A total of five articles comprising 1,883 patients (126 patients with reocclusion, 1,757 patients without reocclusion) who were confirmed to have AIS and who underwent emergency MT were finally included in this study. The pooled analysis (reocclusion versus non-reocclusion) showed that atrial fibrillation [odds ratio (OR), 0.36; 95\% confidence interval (CI), 0.20-0.63], cardiogenic embolism (OR, 0.35; 95\% CI, 0.20-0.63), long-term statin use (OR, 0.39; 95\% CI, 0.21-0.75), long-term antiplatelet use (OR, 0.53; 95\% CI, 0.31-0.92), and target occlusion at middle cerebral artery-M1 (MCA-M1) (OR, 0.39; 95\% CI, 0.19-0.77) might prevent reocclusion and longer onset-to-reperfusion time (mean difference, 66.51; 95\% CI, 36.6696.35) might promote reocclusion after MT performance. Furthermore, the clinical outcomes including early neurological deterioration (OR, 4.87; 95\% CI, 2.08-11.40), 90-day modified Rankin Scale score $\leq 2$ (OR, 0.28; 95\% CI, 0.18-0.45), and 90-day death rate (OR, 1.85; 95\% CI, 1.04-3.29) were also associated with reocclusion after $\mathrm{MT}$ performance.

Conclusions: Atrial fibrillation, cardiogenic embolism, long-term statin use, long-term antiplatelet use, and target occlusion at MCA-M1 might prevent reocclusion, and longer onset-to-reperfusion time seemed to promote reocclusion after MT. Reocclusion after MT results in a high risk of poor prognosis.

Keywords: Thrombectomy; reocclusion; stroke; meta-analysis

Submitted Apr 21, 2020. Accepted for publication Sep 25, 2020.

doi: $10.21037 /$ atm-20-3465

View this article at: http://dx.doi.org/10.21037/atm-20-3465 


\section{Introduction}

As the DAWN (Clinical Mismatch in the Triage of Wake Up and Late Presenting Strokes Undergoing Neurointervention With Trevo) and DEFUSE 3 (Endovascular Therapy Following Imaging Evaluation for Ischemic Stroke 3) trials have been published, mechanical thrombectomy (MT) has become a well-accepted treatment modality for acute ischemic stroke (AIS) $(1,2)$. Despite the high incidence of successful recanalization of over $80 \%$ after MT, 3-9\% of patients display reocclusion in the recanalized vessels within 24 hours after performing MT, imposing a significant impact on people's well-being and quality of life (3-5). Some previous studies have investigated the predictors and prognosis of reocclusion after MT; however, these studies yield conflicting results $(3,6-12)$. We aimed to summarize the existing knowledge and perform a secondary analysis to determine the predictors and prognosis of reocclusion after MT. We presented the following article in accordance with the Preferred Reporting Items for Systematic Reviews and Meta-Analyses (PRISMA) reporting checklist (13) (available at http://dx.doi.org/10.21037/atm20-3465).

\section{Methods}

The subject term "thrombectomy" was searched in several literature databases, including PubMed, Embase, and Cochrane, for publications that were published prior to March 2020. The references of the retrieved articles were also reviewed for additional reports to avoid missing out in our search. The enrolled studies should be screened with the following criteria: (I) patients with AIS and undergoing emergency MT; (II) detailed risk factors and/or clinical outcomes for patients with and/or without reocclusion; and (III) study design that includes retrospective observations and post-hoc analysis for prospective studies. The Newcastle-Ottawa Scale (NOS) was used to evaluate the quality of the enrolled studies $(14,15)$.

\section{Statistical analysis}

R software (http://www.r-project.org) was used for analysis in this study. The values are expressed as mean \pm standard deviation for the continuous data and as number (percentage, \%) for the categorical data. Pooled analysis was performed with the fixed-effects model using the Mantel-Haenszel method if the heterogeneity was expected to be available $\left(\mathrm{I}^{2} \leq 50 \%\right)$. Otherwise, the random-effects model computed by the DerSimonian-Laird method was used $\left(\mathrm{I}^{2}>50 \%\right)$. The pooled results were presented as mean difference (MD) followed by $95 \%$ confidence interval (95\% CI) for the continuous data and as odds ratio (OR) with $95 \%$ CI for the categorical data. All $\mathrm{P}$ values $<0.05$ were considered statistically significant. Publication bias was processed using a funnel plot (RevMan5.0 software). Subgroup analysis was recommended to decrease heterogeneity.

For each of the cases lacking of required data, following calculations may be offset:

(I) When only median (M), interquartile range (IQR), and the sample size were given, as long as the populations was large enough $(\mathrm{n}>25), \mathrm{m}=\mathrm{M}$ and SD $=\mathrm{IQR} / 1.35$;

(II) Given the Group 1 of $\mathrm{n}_{1}, \mathrm{~m}_{1}$ and $\mathrm{SD}_{1}$ and Group 2 of $\mathrm{n}_{2}, \mathrm{~m}_{2}$ and $\mathrm{SD}_{2}$,

(i) total m $=\left(\mathrm{n}_{1} \times \mathrm{m}_{1}+\mathrm{n}_{2} \times \mathrm{m}_{2}\right) /\left(\mathrm{n}_{1}+\mathrm{n}_{2}\right)$;

(ii) total SD $=\left\{\left[\left(\mathrm{n}_{1}-1\right) \times \mathrm{SD}_{1}{ }^{2}+\left(\mathrm{n}_{2}-1\right) \times \mathrm{SD}_{2}{ }^{2}+(\right.\right.$ $\left.\left.\mathrm{n}_{1} \times \mathrm{n}_{2}\right) \times\left(\mathrm{m}_{1}^{2}+\mathrm{m}_{2}^{2}-2 \times \mathrm{m}_{1} \times \mathrm{m}_{2}\right) /\left(\mathrm{n}_{1}+\mathrm{n}_{2}\right)\right] /\left(\mathrm{n}_{1}+\right.$ $\left.\left.\mathrm{n}_{2}-1\right)\right\} 1 / 2$.

\section{Results}

Up to March 2020, a total of 7,230 articles with the subject term "thrombectomy" were identified in the initial research. After screening with the titles and abstracts and thoroughly reading some articles' whole contents, only five articles that met the inclusion criteria were finally included in this meta-analysis. There were a total of 1883 patients who were confirmed to have AIS and underwent emergency MT in the involved studies, in whom 126 patients $(6.69 \%$, $6.59 \%$ within 48 hours) experienced reocclusion after MT and 1,757 patients $(93.31 \%)$ displayed non-reocclusion observed in the follow-up angiography. The mean age was 67.8 years $(62.7$ years in reocclusion and 68.2 years in non-reocclusion). All of the patients had successful MT recanalization (modified Thrombolysis in Cerebral Ischemia, mTICI 2b-3), except for one study (12), where 7 patients had TICI scores of 0. Transcranial Doppler (TCD), magnetic resonance angiography (MRA), and computed tomography angiography (CTA) were performed to determine the reocclusion within 48 hours, except in two reocclusion cases that were followed up between 1 and 180 days in one study (12). Three out of the 5 articles were retrospective studies based on a prospective cohort $(8,10,12)$, 1 was a post-hoc analysis for a prospective cohort (9), and 
Table 1 The characteristics of the enrolled studies

\begin{tabular}{|c|c|c|c|c|c|}
\hline Characteristics & $\begin{array}{l}\text { Hernández-Fernández } \\
\text { et al. }\end{array}$ & Li et al. & Marto et al. & Millán et al. & Mosimann et al. \\
\hline \multicolumn{6}{|l|}{ Demographic } \\
\hline Reocclusion (\%) & $10(25.0)$ & $44(7.2)$ & $28(6.6)$ & $28(29.5)$ & $16(2.3)$ \\
\hline \multicolumn{6}{|l|}{ Interventions } \\
\hline $\begin{array}{l}\text { Recanalization } \\
\text { definitions }\end{array}$ & $\mathrm{TICl} 2 \mathrm{c}-3$ & $\mathrm{mTICl} 2 \mathrm{~b}-3$ & $\mathrm{mTICl} 2 \mathrm{~b}-3$ & $\mathrm{mTICI} 2 \mathrm{~b}-3$ & $\mathrm{mTICl} 2 \mathrm{~b}-3$ \\
\hline \multicolumn{6}{|l|}{ Study features } \\
\hline Design & $\begin{array}{l}\text { Retrospective study } \\
\text { based on a prospective } \\
\text { cohort }\end{array}$ & $\begin{array}{l}\text { Retrospective } \\
\text { study }\end{array}$ & $\begin{array}{l}\text { Retrospective study based } \\
\text { on a prospective cohort }\end{array}$ & $\begin{array}{l}\text { Post-hoc analysis for } \\
\text { a prospective cohort }\end{array}$ & $\begin{array}{l}\text { Retrospective } \\
\text { study based on a } \\
\text { prospective cohort }\end{array}$ \\
\hline NOS & 7 & 8 & 8 & 9 & 8 \\
\hline
\end{tabular}

*, the authors definite reocclusion as AOL 0-1 scores actually, however the baseline data are only presented in the patients with AOL 0-2 scores; due to all of the enrolled patients have complete recanalization after MT, the AOL $0-2$ scores are deemed as reocclusion in this meta-analysis.

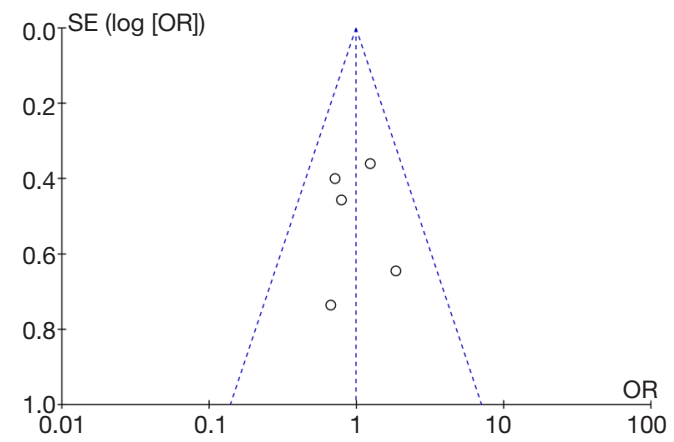

Figure 1 Publication bias from the involved studies.

1 was a retrospective observational study only (11). The publications' NOS score was $\geq 7$, indicating high quality of these studies (15). The aforementioned results are shown in Table 1. The symmetrical graph in the funnel plot indicated that the publication bias from these involved studies was low (Figure 1).

The pooled analysis (reocclusion versus non-reocclusion) showed that atrial fibrillation (OR, 0.36; $95 \% \mathrm{CI}$, 0.20-0.63), cardiogenic embolism (OR, 0.35; 95\% CI, $0.20-0.63)$, long-term statin use (OR, 0.39; 95\% CI, $0.21-$ $0.75)$, long-term antiplatelet use (OR, 0.53; 95\% CI, $0.31-$ 0.92 ), and target occlusion at middle cerebral artery-M1 (MCA-M1) (OR, 0.39; 95\% CI, 0.19-0.77) might prevent reocclusion and longer onset-to-reperfusion time (MD, 66.51; 95\% CI, 36.66-96.35) might promote reocclusion after MT performance (shown in Table 2). Furthermore, the clinical outcomes including early neurological deterioration (the NIHSS scores increased by $\geq 4$ points within 24-48 hours) (OR, 4.87; 95\% CI, 2.08-11.40), 90-day modified Rankin Scale (mRS) score $\leq 2$ (OR, 0.28; $95 \%$ CI, 0.18-0.45), and 90-day death rate (OR, 1.85; 95\% CI, 1.04-3.29) were also associated with reocclusion after undergoing MT. The pooled results are presented in Table 3. 
Table 2 Pooled analysis of the risk factors for reocclusion after MT

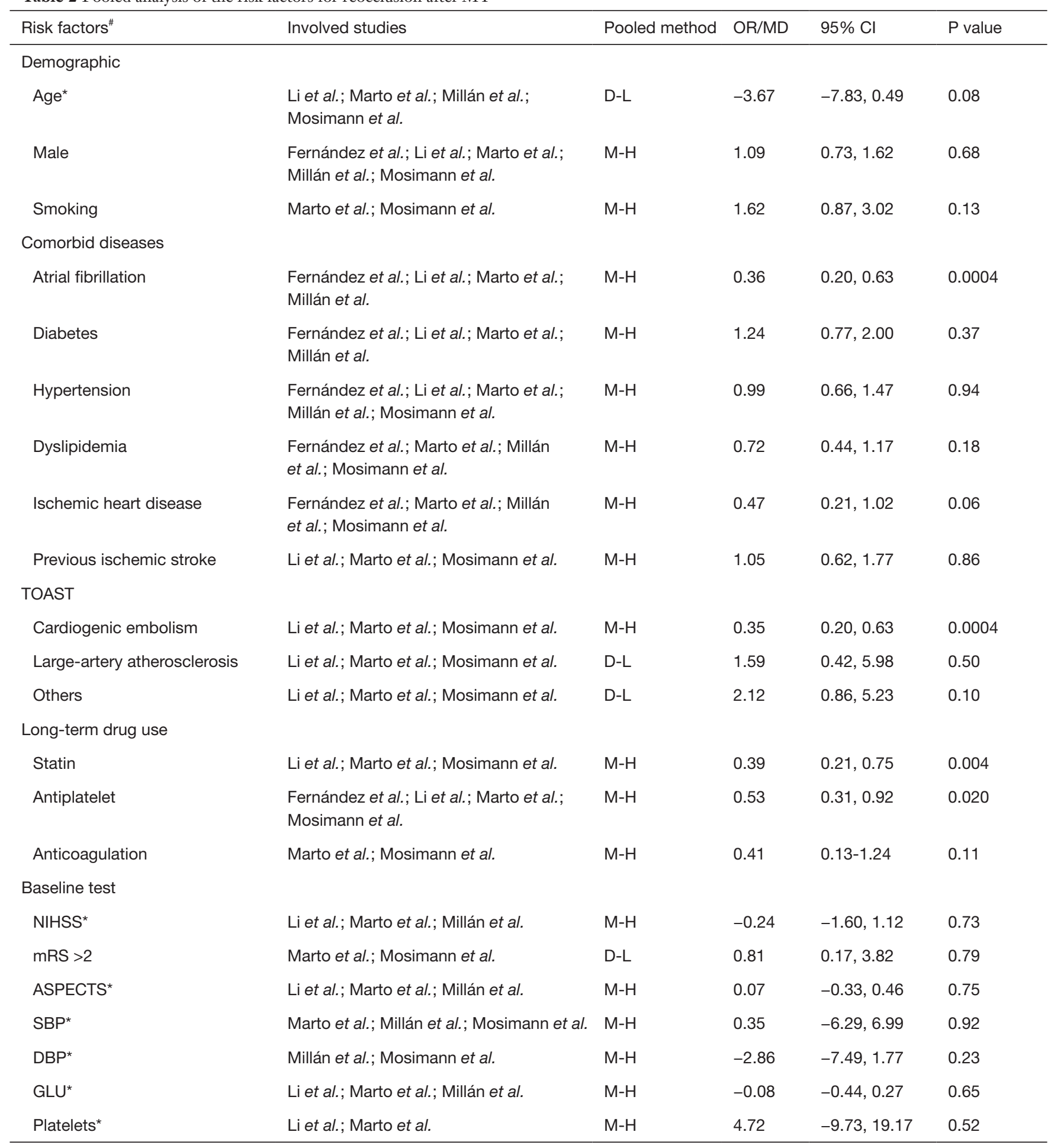

Table 2 (continued) 
Table 2 (continued)

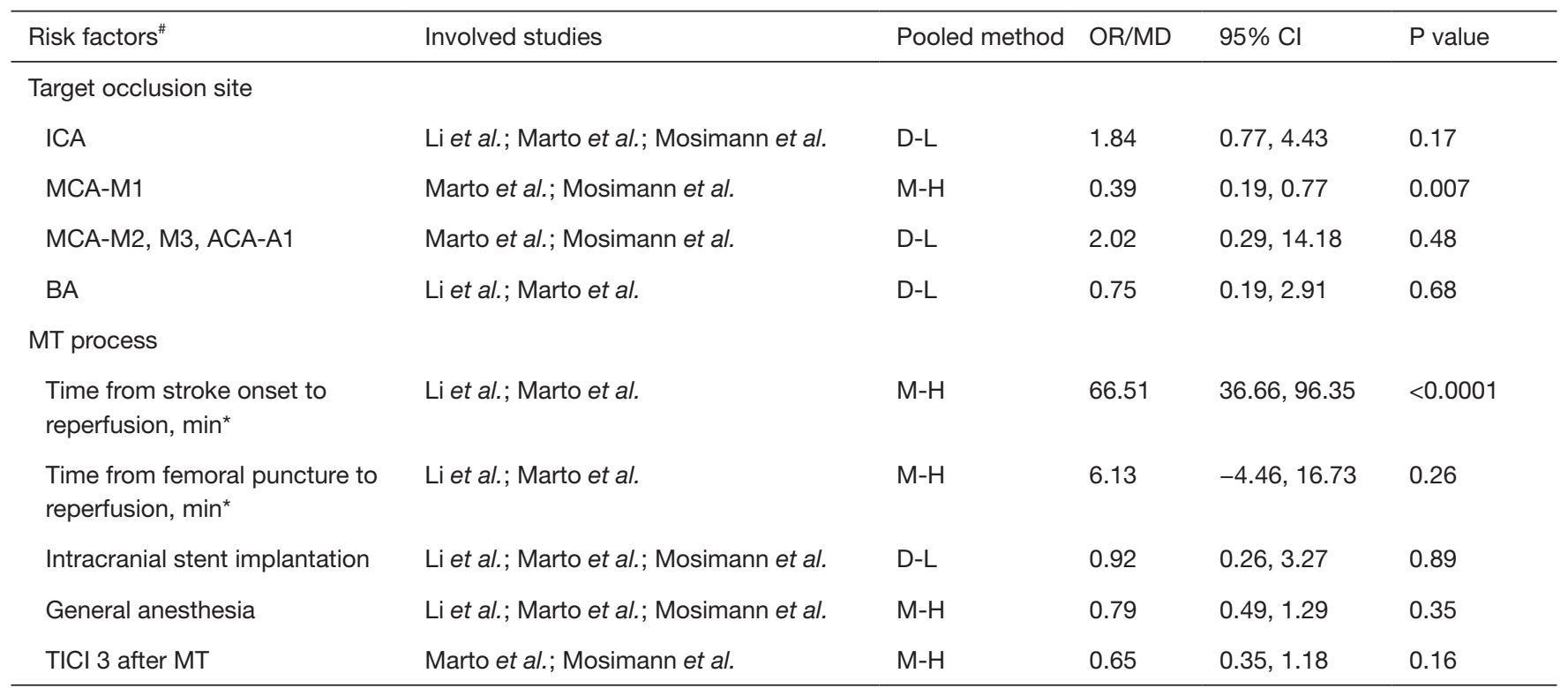

\#, reocclusion vs. non-reocclusion; *, presented as MD. M-H, Mante-Haenszel; D-L, DerSimonian-Laird; MT, thrombectomy; SBP, systolic blood pressure; DBP, diastolic blood pressure; GLU, glucose; ICA, internal carotid artery; MCA, middle cerebral artery; BA, basilar artery.

Table 3 Pooled analysis of the outcomes for the patients with reocclusion $v s$. non-reocclusion after MT

\begin{tabular}{|c|c|c|c|c|c|}
\hline Outcomes & Involved studies & Pooled method & OR & $95 \% \mathrm{Cl}$ & $P$ value \\
\hline 24-hour sICH & Li et al.; Marto et al. & $\mathrm{M}-\mathrm{H}$ & 1.09 & $0.42,2.81$ & 0.85 \\
\hline Early neurological deterioration & Li et al.; Marto et al. & L-D & 4.87 & $2.08,11.40$ & 0.0003 \\
\hline 90 -day $\mathrm{mRS} \leq 2$ & $\begin{array}{l}\text { Li et al.; Marto et al.; Millán et al.; } \\
\text { Mosimann et al. }\end{array}$ & $\mathrm{M}-\mathrm{H}$ & 0.28 & $0.18,0.45$ & $<0.0001$ \\
\hline
\end{tabular}

M-H, Mante-Haenszel; D-L, DerSimonian-Laird; Early neurological deterioration indicates the NIHSS scores increase by $\geq 4$ points within 24-48 hours; MT, mechanical thrombectomy; ICH, intracranial hemorrhage; sICH, symptomatic intracranial hemorrhage.

Considering the high risk of bias for the study conducted by Hernández-Fernández et al., a sensitive analysis was also performed after ruling out these data. The OR and 95\% CI of the seven factors changed when performing the sensitivity analysis (male sex, atrial fibrillation, diabetes, hypertension, dyslipidemia, ischemic heart disease, and long-term antiplatelet use), with atrial fibrillation (OR, $0.33 ; 95 \%$ CI, 0.19-0.60) and long-term antiplatelet use (OR, 0.49; 95\% CI, 0.28-0.87) being also associated with reocclusion after MT. All the aforementioned results are shown in Table 4.

\section{Discussion}

This secondary analysis further identified that the postMT reocclusion-related factors included atrial fibrillation, cardiogenic embolism, long-term statin use, long-term antiplatelet use, target occlusion at MCA-M1, and onset-toreperfusion time. Reocclusion predicted higher possibilities of early neurological deterioration and 90-day death and a lower possibility of 90 -day $\mathrm{mRS}$ score $\leq 2$.

As recommended by the latest American Heart Association/American Stroke Association guidelines and clinical trials, MT with or without intravenous thrombolysis 
Table 4 Sensitive analysis of the risk factors for reocclusion after MT

\begin{tabular}{|c|c|c|c|c|c|}
\hline Risk factors ${ }^{\#}$ & Involved studies & Pooled method & OR & $95 \% \mathrm{Cl}$ & $P$ value \\
\hline \multicolumn{6}{|l|}{ Demographic } \\
\hline Male & $\begin{array}{l}\text { Li et al.; Marto et al.; Millán et al.; Mosi- } \\
\text { mann et al. }\end{array}$ & $\mathrm{M}-\mathrm{H}$ & 1.16 & $0.76,1.75$ & 0.49 \\
\hline \multicolumn{6}{|l|}{ Comorbid diseases } \\
\hline Hypertension & $\begin{array}{l}\text { Li et al.; Marto et al.; Millán et al.; Mosi- } \\
\text { mann et al. }\end{array}$ & $\mathrm{M}-\mathrm{H}$ & 1.02 & $0.67,1.55$ & 0.93 \\
\hline Dyslipidemia & Marto et al.; Millán et al.; Mosimann et al. & $\mathrm{M}-\mathrm{H}$ & 0.80 & $0.48,1.33$ & 0.38 \\
\hline
\end{tabular}

\#, reocclusion vs. non-reocclusion. M-H, Mante-Haenszel; D-L, DerSimonian-Laird; MT, thrombectomy.

was the cornerstone for treating AIS in emergency cases (16). However, reocclusion can be encountered during MT of acute large-vessel occlusions, which may significantly deteriorate patients' neurological functions (16). Theoretically, endothelial damage is the major pathogenesis of reocclusion $(6,7,17,18)$. The vascular endothelium can be easily injured during MT, causing reocclusion. Abraham et al. revealed the endothelial injury in the reocclusion site using magnetic resonance imaging, in which the signal enhancement of the vessel wall that represents the endothelial injury can be clearly observed (17). Additionally, other factors contributing to thrombogenesis in the recanalized vessels after MT mainly include platelet aggregation, tissue factor release, and inflammation (18). These pathogenic mechanisms are possibly consistent with the clinical findings in this meta-analysis.

This meta-analysis totally incorporated 5 articles, all of which were retrospective case-control studies. Despite the lack of high-quality evidence, considering the large sample size and a wide array of involved factors in our analysis, more comprehensive results were obtained. Meanwhile, the high quality of the involved studies (NOS score $\geq 7$ ) enhanced the power of the test. Therefore, compared to previous publications, our pooled study provided a more convincing conclusion for the first time. Considering the recommendation from the latest guidelines, MT was considered as the primary strategy for treating AIS. Other endovascular therapies such as intra-arterial thrombolysis and mechanical thrombus disruption were only used as adjuvant approaches (16). As a result, the articles mainly focusing on other endovascular therapies, rather than MT, were removed in this study. It is noteworthy that some method bias is inevitable in these involved studies. The definition of reocclusion is unclear in 3 articles, all of which only declares that reocclusion is detected with TCD, MRA, or CTA $(8,10,11)$. In contrast, the other 2 articles detail the quantitative methods for identifying reocclusion $(9,12)$. This may further produce information bias. Similarly, all of the enrolled patients displayed successful MT recanalization, which were detected with an imaging test within 24-48 hours, except for 7 patients displaying nonrecanalization after MT (TICI score, 0) and 2 patients determining the reocclusion at the long-term follow-up (Hernández-Fernández et al.) (12). Despite the risk of bias among these articles, a low percentage of heterogeneous cases may have little impact on our final results.

Although we showed that $6.59 \%$ of the patients experienced reocclusion after MT, there was a significant discrepancy in the reocclusion rates after MT among the previous publications (2.3-29.5\%), which may be due to varying baseline characteristics among these cohorts (8-12). Therefore, this study aimed to investigate the correlated factors of reocclusion based on these datasets to subsequently increase the odds of favorable outcomes. According to the pooled analysis, we found that atrial fibrillation, cardiogenic embolism, long-term statin use, long-term antiplatelet use, and target occlusion in MCA-M1 might prevent reocclusion occurrence after MT. 
Furthermore, the onset-to-reperfusion time was positively associated with reocclusion after MT. It has been fully corroborated that in situ thrombosis plays an essential role in reocclusion after MT (6). Endovascular injury is capable of inducing platelet aggregation and adhesion, promoting coagulation activities, and producing inflammation factors, ultimately causing thrombotic occlusion (18). Our findings exactly corresponded with the pathogenesis. Cardiogenic embolism (including atrial fibrillation) is the major cause of exogenous embolism; thus, the target occlusion is more likely induced by embolism than in situ thrombosis (16). This indicates that the endothelium is relatively intact at the target occlusion site; therefore, reocclusion after MT is rarely observed. Additionally, embolism is also the main cause of acute MCA-M1 occlusion, whereas in situ thrombosis induced by atherosclerosis always leads to chronic MCA-M1 stenosis. The target occlusion site at MCA-M1 may also be less likely to display reocclusion after MT. Long-term statin and antiplatelet treatment can prevent platelet aggregation and suppress vascular inflammation, which may exert protective effects on vascular endothelium and, as a result, prevent thrombogenesis after MT. Moreover, longer onset-to-reperfusion time indicates more severe endovascular injury; thus, the target vessels are more susceptible to reocclusion after MT.

Consistent with previous studies, based on the current study, reocclusion after MT had a poor prognosis. The pooled results revealed that patients with reocclusion after MT displayed higher rates of early neurological deterioration and 90-day death and a lower rate of 90day $\mathrm{mRS}$ score $\leq 2$ than patients without reocclusion. Repeated reperfusion and occlusion at the target site may aggravate ischemia-reperfusion injury and oxidative stress injury, leading to severe brain infarction (19). Because of the high risk of bias in the study conducted by HernándezFernández et al., we removed this study in our sensitive analysis (12). The correlated factors for reocclusion after MT were unchanged, which further supported our results.

This study has several limitations. First, our metaanalysis only included retrospective studies, and the inevitable bias in the retrospective studies might have an unexpected impact on our overall conclusions. As mentioned above, information bias and selective bias could be observed in these studies, which may bias our results toward the null hypothesis.

In summary, this meta-analysis suggested that atrial fibrillation, cardiogenic embolism, long-term statin use, long-term antiplatelet use, and target occlusion at
MCA-M1 might prevent reocclusion and longer onsetto-reperfusion time seemed to promote reocclusion after MT. Reocclusion after MT results in a high risk of poor prognosis. Considering the limitations of this study, further large cross-sectional studies are required to draw more reliable conclusions.

\section{Acknowledgments}

We would like to thank all patients and doctors who participated in this study for their cooperation.

Funding: None.

\section{Footnote}

Reporting Checklist: The authors have completed the PRISMA reporting checklist. Available at http://dx.doi. org/10.21037/atm-20-3465

Conflicts of Interest: All authors have completed the ICMJE uniform disclosure form (available at http://dx.doi. org/10.21037/atm-20-3465). JD serves as an unpaid section editor of Annals of Translational Medicine currently. The other authors have no conflicts of interest to declare.

Ethical Statement: The authors are accountable for all aspects of the work in ensuring that questions related to the accuracy or integrity of any part of the work are appropriately investigated and resolved.

Open Access Statement: This is an Open Access article distributed in accordance with the Creative Commons Attribution-NonCommercial-NoDerivs 4.0 International License (CC BY-NC-ND 4.0), which permits the noncommercial replication and distribution of the article with the strict proviso that no changes or edits are made and the original work is properly cited (including links to both the formal publication through the relevant DOI and the license). See: https://creativecommons.org/licenses/by-nc-nd/4.0/.

\section{References}

1. Nogueira RG, Jadhav AP, Haussen DC, et al. Thrombectomy 6 to 24 Hours after Stroke with a Mismatch between Deficit and Infarct. N Engl J Med 2018;378:11-21.

2. Albers GW, Marks MP, Kemp S, et al. Thrombectomy for Stroke at 6 to 16 Hours with Selection by Perfusion 


\section{Page 8 of 8}

Imaging. N Engl J Med 2018;378:708-18.

3. Zhao W, Shang S, Li C, et al. Long-term outcomes of acute ischemic stroke patients treated with endovascular thrombectomy: A real-world experience. J Neurol Sci 2018;390:77-83.

4. Janjua N, Alkawi A, Suri MF, et al. Impact of arterial reocclusion and distal fragmentation during thrombolysis among patients with acute ischemic stroke. AJNR Am J Neuroradiol 2008;29:253-8.

5. Tsang ACO, Orru E, Klostranec JM, et al. Thrombectomy Outcomes of Intracranial Atherosclerosis-Related Occlusions. Stroke 2019;50:1460-6.

6. Kang DH, Kim YW, Hwang YH, et al. Instant reocclusion following mechanical thrombectomy of in situ thromboocclusion and the role of low-dose intra-arterial tirofiban. Cerebrovasc Dis 2014;37:350-5.

7. Hidaka M, Yamaguchi S, Koyanagi Y, et al. Reocclusion of the treated vessel due to endothelial injury after mechanical thrombectomy in a patient with acute ischaemic stroke. BMJ Case Rep 2019;12:e228937.

8. Mosimann PJ, Kaesmacher J, Gautschi D, et al. Predictors of Unexpected Early Reocclusion After Successful Mechanical Thrombectomy in Acute Ischemic Stroke Patients. Stroke 2018;49:2643-51.

9. Millán M, Remollo S, Quesada H, et al. Vessel Patency at 24 Hours and Its Relationship With Clinical Outcomes and Infarct Volume in REVASCAT Trial (Randomized Trial of Revascularization With Solitaire FR Device Versus Best Medical Therapy in the Treatment of Acute Stroke Due to Anterior Circulation Large Vessel Occlusion Presenting Within Eight Hours of Symptom Onset). Stroke 2017;48:983-9.

10. Marto JP, Strambo D, Hajdu SD, et al. Twenty-four-hour reocclusion after successful mechanical thrombectomy: associated factors and long-term prognosis. Stroke 2019;50:2960-3.

Cite this article as: $\mathrm{Li} \mathrm{X,} \mathrm{Gu} \mathrm{F,} \mathrm{Ding} \mathrm{J,} \mathrm{Bian} \mathrm{J,} \mathrm{Wang} \mathrm{N,}$ Shu R, Li Q, Xu X. The predictors and prognosis for unexpected reocclusion after mechanical thrombectomy: a meta-analysis. Ann Transl Med 2020;8(23):1566. doi: 10.21037/ atm-20-3465
Li et al. Predictors and prognosis for reocclusion after thrombectomy

11. Li W, Ding J, Sui X, et al. Prognosis and risk factors for reocclusion after mechanical thrombectomy. Ann Clin Transl Neurol 2020;7:420-8.

12. Hernández-Fernández F, Del Valle Pérez JA, GarcíaGarcía J, et al. Simultaneous Angioplasty and Mechanical Thrombectomy in Tandem Carotid Occlusions. Incidence of Reocclusions and Prognostic Predictors. J Stroke Cerebrovasc Dis 2020;29:104578.

13. Moher D, Liberati A, Tetzlaff J, et al. Preferred reporting items for systematic reviews and meta-analyses: the PRISMA statement. Int J Surg 2010;8:336-41.

14. Higgins JP, Altman DG, Gøtzsche PC, et al. The Cochrane Collaboration's tool for assessing risk of bias in randomised trials. BMJ 2011;343:d5928.

15. Wells GA, Shea B, O'Connell D, et al. The NewcastleOttawa Scale (NOS) for assessing the quality of nonrandomized studies in metaanalyses. Ottawa Hospital Research Institute 2013 Available online: http://www.ohri. ca/programs/clinical_epidemiology/oxford.htm

16. Powers WJ, Derdeyn CP, Biller J, et al. 2015 American Heart Association/American Stroke Association Focused Update of the 2013 Guidelines for the Early Management of Patients With Acute Ischemic Stroke Regarding Endovascular Treatment: A Guideline for Healthcare Professionals From the American Heart Association/ American Stroke Association. Stroke 2015;46:3020-35.

17. Abraham P, Scott Pannell J, Santiago-Dieppa DR, et al. Vessel wall signal enhancement on 3-T MRI in acute stroke patients after stent retriever thrombectomy. Neurosurg Focus 2017;42:E20.

18. Becker R. Dynamics of coronary thrombolysis and reocclusion. Clin Cardiol 1997;20:III2-5.

19. Dewhurst TA, Bruneau R, Titus B, et al. Percutaneous rotational thrombectomy reduces acute reocclusion in an animal model of acute coronary thrombosis. Cathet Cardiovasc Diagn 1993;30:120-6. 